\title{
Creating a Local Geographic Influenza-like IIIness Activity Report
}

\author{
Dino Rumoro ${ }^{1}$, Shital Shah 1 , Gordon Trenholme', Gillian Gibbs ${ }^{* 1}$, Marilyn Hallock ${ }^{1}$ and \\ Michael J. Waddell ${ }^{2}$
}

${ }^{1}$ Emergency Medicine, Rush University Medical Center, Chicago, IL, USA; ${ }^{2}$ Pangaea Information Technologies, Chicago, IL, USA

\section{Objective}

To create a local geographic influenza-like illness (ILI) activity report.

\section{Introduction}

Mapping ILI surveillance data can be useful in identifying the direction and speed of an outbreak and for focusing control measures for an efficient public health response. The Centers for Disease Control and Prevention's (CDC) ILINet currently displays weekly ILI geographic data at a national/regional/state level, but this visual data could also be useful at the local level.

\section{Methods}

The data elements that were included in this study were: daily emergency department (ED) ILI rate, 7 day moving average ED ILI rate, zip code, and date from October 1, 2013 to March 31, 2014. The data region was the catchment area of an urban academic medical center (AMC). The data were processed using the GUARDIAN (Geographic Utilization of Artificial Intelligence in Real-Time for Disease Identification and Alert Notification) surveillance system. We created the following derived variables in percentages:

- ILI within a zip code = ILI positive ED visits within zip code/total ED visits within zip code

- Proportion of zip code ILI with regional ILI = ILI positive ED visits within zip code/ total ILI positive ED visits within the AMC region

- Zip code representation = total ED visits within zip code/total ED visits within the $\mathrm{AMC}$ region

These three variables provide information on the relative importance of the ILI rate within a zip code compared to the overall ILI rate within the AMC region. These trends were utilized to generate rules for the development of a color coded zip code map, as follows:

- White represents no data available or $0 \%$ of total ED visits within the AMC region are in the zip code

- Gray represents insufficient data or $<10 \%$ and $>0 \%$ of total ED visits within the $\mathrm{AMC}$ region are in the zip code

- Green represents within normal limits and requires $\geq 10 \%$ of total ED visits within the AMC region are in the zip code AND ILI rate is below $10 \%$ within the zip code

- Yellow represents more than 2 standard deviations above the mean and requires $\geq 10 \%$ of total $\mathrm{ED}$ visits within the $\mathrm{AMC}$ region are in the zip code AND ILI rate is between $10 \%$ and $12.5 \%$ within the zip code

- Orange represents more than $20 \%$ of all positive ILI cases come from that zip code within the AMC region

- Red represents more than 3 standard deviations above the mean and requires $\geq 10 \%$ of total $\mathrm{ED}$ visits within the $\mathrm{AMC}$ region are in the zip code AND ILI rate is above $12.5 \%$ within the zip code

The thresholds utilized in the rules were either generated through historical statistical analysis or based on the need for an adequate sample size. The rules were translated into the GUARDIAN system, which auto-generates daily reports of geographic ILI activity at the AMC.

\section{Results}

Each morning, the GUARDIAN reporting module generates a map for the AMC's current 7-day moving average ILI rate by zip code (Figure 1). The resulting map and a summary table are combined into a PDF document and emailed out to the response team at the AMC. In addition, an interactive web-based version of the map is made available through the GUARDIAN user interface, which allows clinicians to review individual patient's charts.

\section{Conclusions}

The ILI activity report provides zip code level geographic analysis of ILI prevalence using the developed color scheme. With additional data, such as multiple ED sites and temporal information, the ILI activity maps could be further enhanced to capture spatial/temporal changes in ILI rates within the broader region (e.g., metropolitan area, state level, or CDC region). These daily auto-generated geographic reports can be utilized by clinical and public health personnel for monitoring ILI activity within their regions, as well as initiating appropriate emergency management protocols.

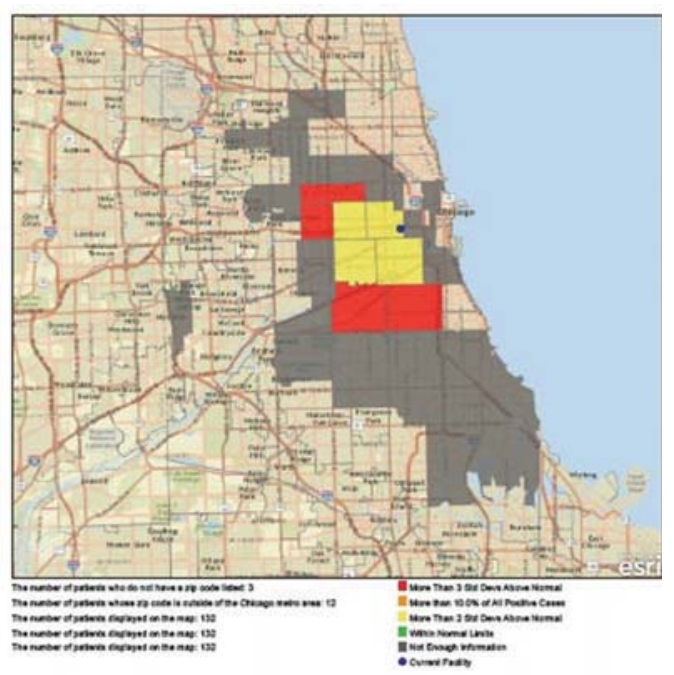

Figure 1: Map of the seven day moving average ILI rate by zip code on January 14, 2014.

\section{Keywords}

Influenza-like illness surveillance; GUARDIAN; GIS

\section{Acknowledgments}

GUARDIAN is funded by the US Department of Defense, Telemedicine and Advanced Technology Research Center, Award numbers W81XWH-09-1-0662 and W81XWH-11-1-0711.

\section{*Gillian Gibbs}

E-mail: Gillian_Gibbs@rush.edu 